\title{
Germacrone inhibits the proliferation of glioma cells by promoting apoptosis and inducing cell cycle arrest
}

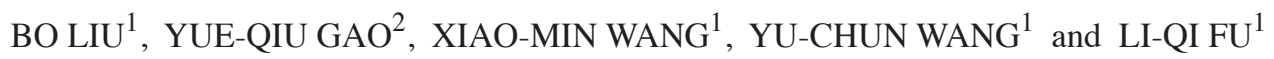 \\ ${ }^{1}$ Department of Neurosurgery, Daqing Oilfield General Hospital, Daqing, Heilongjiang 163411; \\ ${ }^{2}$ Department of Gastroenterology and Hepatology, The Fifth Affiliated Hospital of \\ Harbin Medical University, Daqing, Heilongjiang 163316, P.R. China
}

Received August 16, 2013; Accepted March 6, 2014

DOI: $10.3892 / \mathrm{mmr} .2014 .2290$

\begin{abstract}
Germacrone is one of the major bioactive components of the traditional Chinese Medicinal plant Curcuma aromatica Salisb. and has been shown to possess anti-tumor properties. In the present study, the anti-proliferative effect of germacrone on human glioma cells and the molecular mechanism underlying its cytotoxicity were investigated. Treatment of the U87 and U251 human glioma cell lines with germacrone inhibited the cell proliferation in a dose- and time-dependent manner as assessed by MTT assay, while significantly lower effects were observed on normal human astrocytes. Flow cytometric analysis and DNA fragmentation revealed that germacrone promoted apoptosis of glioma cells, associated with an increased expression of p53 and bax and decreased expression of bcl-2. Furthermore, flow cytometric cell cycle analysis revealed that germacrone induced G1 phase arrest, associated with an obvious decrease in the expression of cyclin D1 and CDK2 and an increased expression of p21. In conclusion, the present study suggested that germacrone may be a novel potent chemopreventive drug candidate for gliomas via regulating the expression of proteins associated with apoptosis and G1 cell cycle arrest.
\end{abstract}

\section{Introduction}

Gliomas are the most common tumor types of the central nervous system with a low 5-year survival rate and high morbidity rate (1). The current clinical treatments for glioma include surgery, radiotherapy and chemotherapy (2). However, accumulating evidence shows that the resistance of glioma cells to conventional drugs is becoming a problem and it is imperative to find novel and effective drugs against glioma (3).

Correspondence to: Dr Bo Liu, Department of Neurosurgery, Daqing Oilfield General Hospital, No. 9 Zhong Kang Street, Daqing, Heilongjiang 163411, P.R. China

E-mail: xlb7657@163.com

Key words: germacrone, glioma cells, apoptosis, cell cycle arrest
Traditional Chinese Medicinal plants are a significant source of drugs that serve as potential therapeutic compounds for the treatment of cancer (4). The rhizome of Curcuma aromatica Salisb. is widely used as a traditional herbal medicine for anti-tumor therapy in China, Japan and other Asian countries (5). Findings of recent studies have demonstrated that germacrone, a major bioactive component of Curcuma aromatica Salisb., possesses anti-tumor, anti-inflammatory and neuroprotective properties. Claeson et al (6) found that germacrone had an obvious anti-inflammatory activity against carrageenan-induced hind paw edema in rats. Matsuda et al (7) and Morikawa et al (8) reported that germacrone exerted a potent protective effect on acute liver injury in mice induced by D-galactosamine/lipopolysaccharide and tumor necrosis factor- $\alpha$. Another study showed that the treatment of HepG2 and Bel7402 hepatoma cell lines with germacrone promoted apoptosis associated with the upregulation of bax and the downregulation of bcl-2. The upregulation of p53 and an increase in reactive oxygen species were observed, which suggested that germacrone is a novel potent drug candidate for liver cancer (9). Furthermore, germacrone was found to inhibit the proliferation of breast cancer cell lines MCF-7 and MDA-MB-231 by inducing cell cycle arrest in the G0/G1 and G2/M phase as well as apoptosis through a mitochondria-mediated caspase pathway (10). However, to the best of our knowledge, this is the first study to investigate the inhibitory effect of germacrone on human glioma cells. Therefore, in the present study, the anti-proliferative effect of germacrone on glioma cells and normal human astrocytes as well as the mechanism of action of the anti-tumor activity were investigated.

\section{Materials and methods}

Germacrone ( $>95 \%$ ) was purchased from Sigma (St. Louis, MO, USA). RPMI-1640 culture medium, Dulbecco's medium Eagle's medium (DMEM), fetal bovine serum (FBS), phosphate-buffered saline (PBS), penicillin-streptomycin (PS) and $0.25 \%(\mathrm{w} / \mathrm{v})$ trypsin/1 mM EDTA were purchased from Gibco (Grand Island, NY, USA).

Cell lines and culture. The U87 and U251 human glioma cell lines were obtained from the Shanghai Institute of Cell Biology, Chinese Academy of Sciences (Shanghai, China). 
Cells were cultured in DMEM supplemented with 10\% FBS, $2 \mathrm{mmol} / \mathrm{l}$ glutamine, penicillin $(100 \mathrm{U} / \mathrm{ml})$ and streptomycin $(100 \mathrm{mg} / \mathrm{ml})$, and maintained at $37^{\circ} \mathrm{C}$ and $5 \% \mathrm{CO}_{2}$ in a humid environment. Normal human astrocytes (NHA) were obtained as part of the human astrocytes kit (Gibco) and cultured in astrocyte medium.

MTT assay. Cell proliferation was evaluated by MTT assay. Briefly, the cells were seeded into 96-well plates at a density of $3 \times 10^{4}$ (cells/well) and left to adhere overnight. The cells were then incubated with or without $0-250 \mu \mathrm{mol} / 1$ germacrone prior to addition of $10 \mathrm{ml}$ of $5 \mathrm{mg} / \mathrm{ml}$ MTT and incubation in the dark at $37^{\circ} \mathrm{C}$ for $2 \mathrm{~h}$. Absorbance was determined at a wavelength of $492 \mathrm{~nm}$.

Flow cytometric analysis. Cells were incubated with germacrone at different concentrations $(0-250 \mu \mathrm{mol} / \mathrm{l})$ for $24 \mathrm{~h}$. The cells were washed with PBS, detached with trypsin and harvested. The cells were resuspended in $1 \mathrm{ml}$ Hoechst 33258 for $5 \mathrm{~min}$ and washed with PBS three times. Apoptotic cells were detected by staining with annexin V-FITC/PI according to the protocol of the annexin V-FITC cell Apoptosis Detection Kit (BD Biosciences, Franklin Lakes, NJ USA).

DNA fragmentation. For DNA laddering, following exposure to drug treatment, $2 \times 10^{6}$ cells were suspended in PBS and homogenized in buffer containing proteinase $\mathrm{K}$ and RNase. Subsequently, cell lysates were incubated at $50^{\circ} \mathrm{C}$ for $30 \mathrm{~min}$, and isopropranol was added. DNA was precipitated and washed by $70 \%$ ethanol. DNA was then analyzed using $1.2 \%$ agarose gel electrophoresis.

Cell cycle. Cells were seeded at a density of $1.0 \times 10^{6}$ cells and incubated with germacrone at various concentrations (0-250 $\mu \mathrm{mol} / \mathrm{l})$ for $24 \mathrm{~h}$. Following two washes with PBS, the cells were harvested and collected by centrifugation, followed by fixation in ice-cold $70 \%$ ethanol at $-20^{\circ} \mathrm{C}$ overnight. Cells were then collected and stained with $100 \mu \mathrm{l}$ PI solution for $30 \mathrm{~min}$ in the dark followed by cell cycle analysis.

Western blot analysis. Following incubation with germacrone at various concentrations $(0-250 \mu \mathrm{mol} / \mathrm{l})$, total cell lysates were prepared and subjected to SDS-PAGE. For western blot analysis, primary antibodies used included anti-p53, B-cell lymphoma 2 (bcl-2), bcl-2 associated X (bax), cyclin D1 and cyclin D kinase 2 (CDK2) (Cell Signaling, Beverly, MA, USA) as well as p21 and GAPDH (Santa Cruz Biotechnology, Inc., Santa Cruz, CA, USA). Anti-rabbit or anti-mouse secondary antibody conjugated with horseradish peroxidase was also used (Pierce Chromatography Cartridges, Rockford, IL, USA). Immunoreactive bands were detected using an enhanced chemiluminescence (ECL) kit for western blot analysis and the ChemiGenius bioimaging system (Syngene, Cambridge, UK).

Statistical analysis. Data were presented as the mean \pm standard deviation and processed for statistical analysis by the SPSS program (SPSS, Inc., Chicago, IL, USA). A comparison between groups was made using analysis of variance (ANOVA), and a statistically significant difference between values was defined as $\mathrm{P}<0.05$.
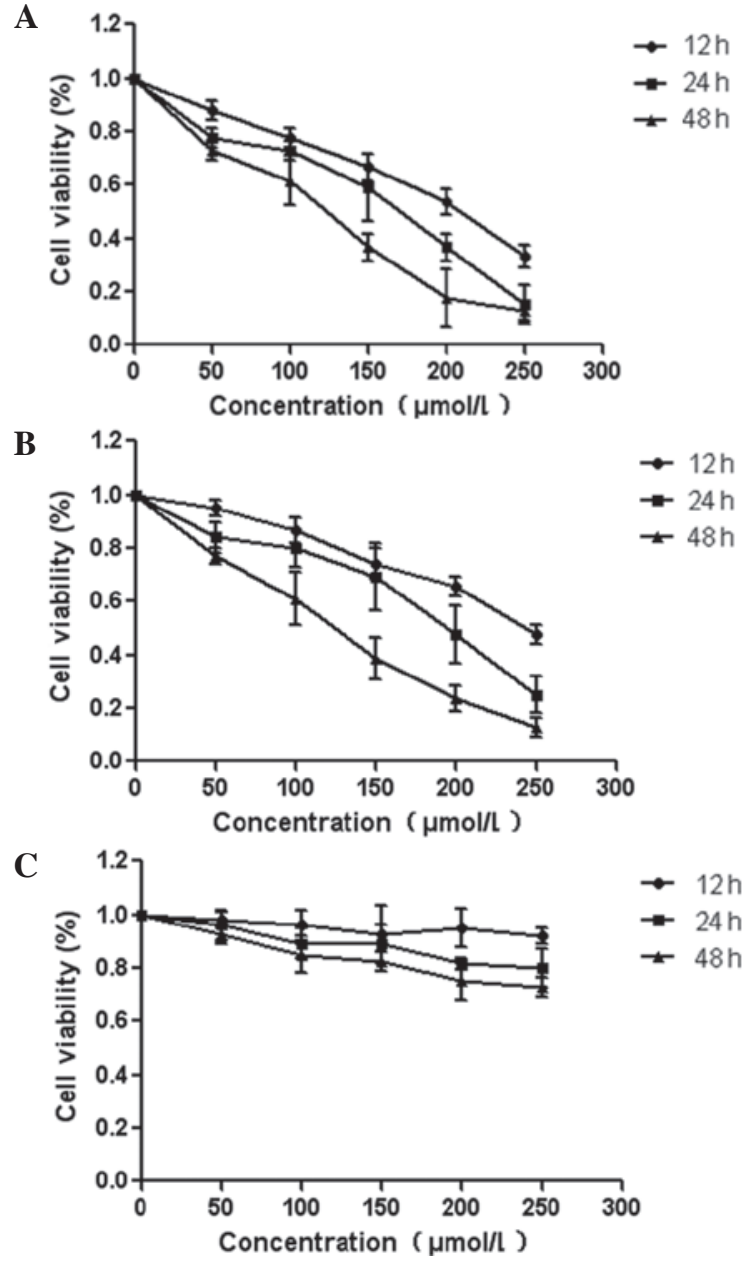

Figure 1. Effect of germacrone on the proliferation of glioma cells and normal human astrocytes. Cells were incubated with germacrone for 12 , 24 and $48 \mathrm{~h}$ at different concentrations $(0-250 \mu \mathrm{mol} / \mathrm{l})$. The proliferation of (A) U87 glioma cell line, (B) U251 glioma cell line and (C) normal human astrocytes was assessed using the MTT assay. ${ }^{*} \mathrm{P}<0.05 ;{ }^{* *} \mathrm{P}<0.01$.

\section{Results}

Germacrone inhibits the proliferation of human glioma cells. To investigate the effects of germacrone on the proliferation of glioma cells, U87 and U251 cells were incubated with $0-250 \mu \mathrm{mol} / 1$ of germacrone for different time periods using the MTT assay. As shown in Fig. 1, germacrone significantly inhibited the proliferation of U87 and U251 cells in a timeand dose-dependent manner. Compared with glioma cells, exposure of normal human astrocytes to germacrone exerted no obvious inhibitory effect as shown in Fig. 1C.

Germacrone induces apoptosis in human glioma cells. The apoptotic effect of germacrone on human glioma cells was investigated by flow cytometry. The U87 and U251 cell lines were incubated with germacrone at different concentrations for $24 \mathrm{~h}$ and the apoptotic rate was analyzed using annexin $\mathrm{V}$ and PI staining. As shown in Fig. 2, treatment with germacrone (50, 150 and $250 \mu \mathrm{mol} / \mathrm{l})$ for $24 \mathrm{~h}$ significantly increased the apoptotic rates of U87 (Fig. 2A) and U251 (Fig. 2B) compared with the control. The DNA fragmentation experiment also showed that germacrone induced the apoptosis of U87 cells following 


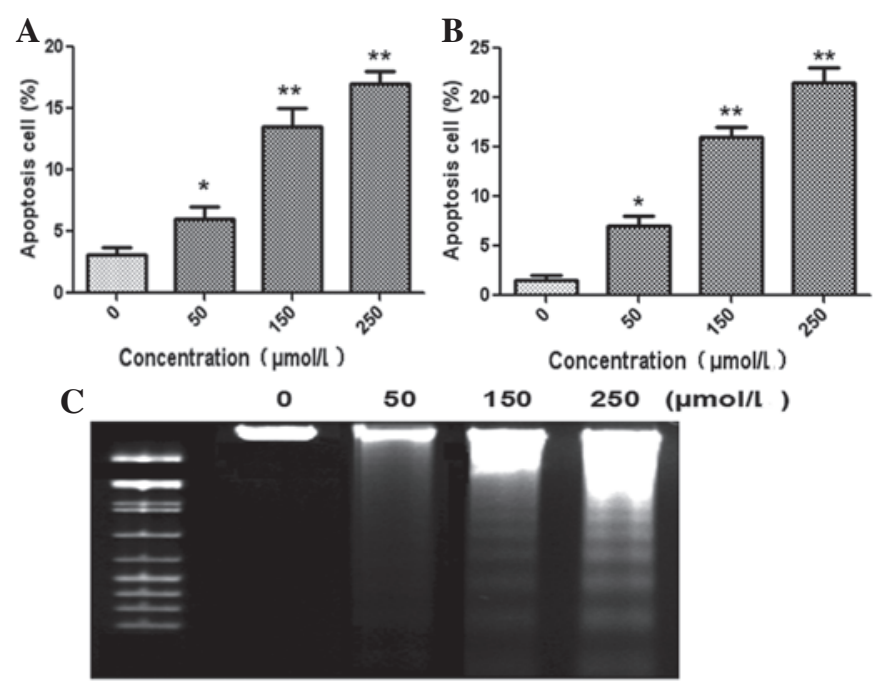

Figure 2. Effect of germacrone on apoptosis of glioma cells. Cells were treated with germacrone at different concentrations $(50,150$ and $250 \mu \mathrm{mol} / \mathrm{l})$ for $24 \mathrm{~h}$. The apoptotic rate of the (A) U87 and (B) U251 human glioma cell lines was detected by flow cytometry. (C) Incubation with different concentrations of germacrone for $24 \mathrm{~h}$ resulted in the fragmented DNA ladder of U87 cells. ${ }^{*} \mathrm{P}<0.05 ;{ }^{* *} \mathrm{P}<0.01$.
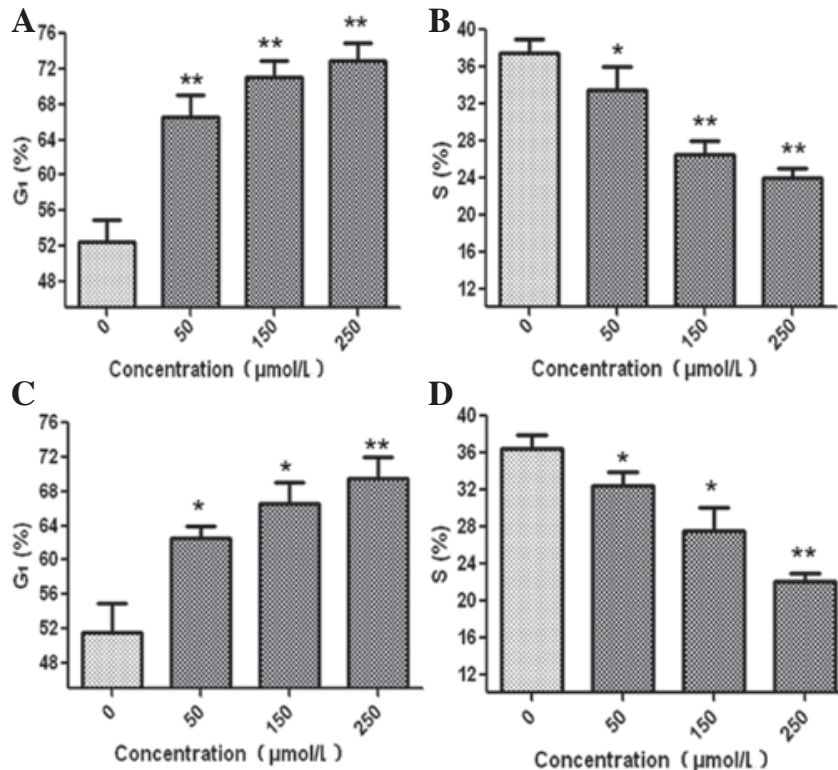

D

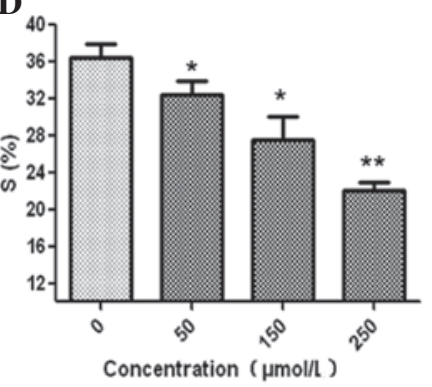

Figure 3. Effect of germacrone on cell cycle arrest in glioma cells. Cells were incubated with germacrone at different concentrations $(50,150$ and $250 \mu \mathrm{mol} / \mathrm{l})$ for $24 \mathrm{~h}$. The cell cycle distribution of U87 and U251 was analyzed by flow cytometry. (A) G1 and (B) S represent the cell cycle phases of U87, while (C) and (D) refer to the cell cycle distribution of $\mathrm{U} 251 .{ }^{*} \mathrm{P}<0.05$; ${ }^{* *} \mathrm{P}<0.01$.

incubation for $24 \mathrm{~h}$ at different concentrations (Fig. 2C). Similar DNA ladders were also observed in U251 cells (data not shown).

Germacrone induces cell cycle arrest in G1 phase in glioma cells. Human glioma cells were treated with germacrone at different concentrations for $24 \mathrm{~h}$ to investigate its effect on the cell cycle distribution. In the U87 human glioma cell line, germacrone significantly increased the proportion of cells in
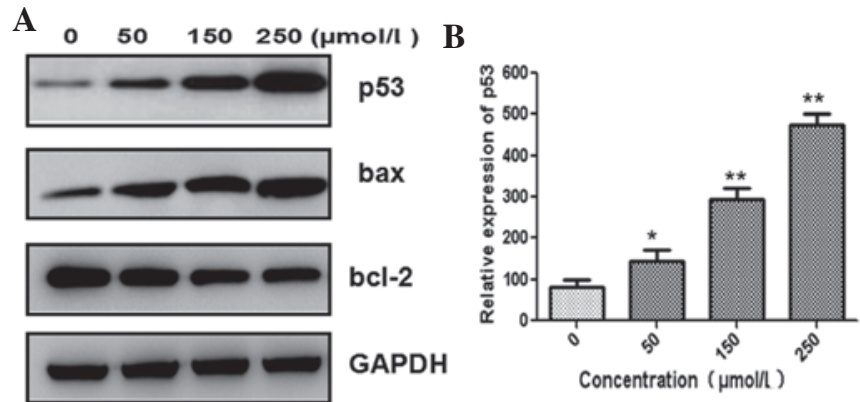

C

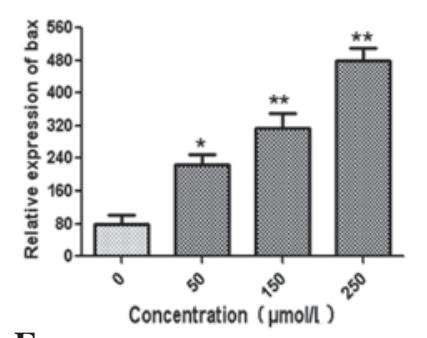

$\mathbf{E}$

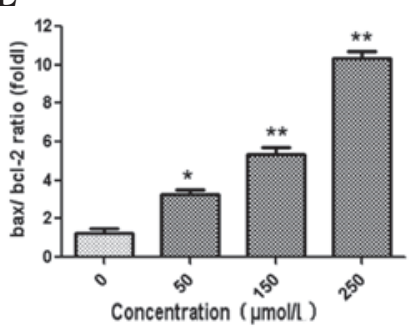

Figure 4. Effect of germacrone on the expression of apoptosis-associated proteins. Glioma cells were incubated with germacrone at different concentrations for $24 \mathrm{~h}$ and subjected to western blot analysis. (A) Expression levels of apoptosis-associated proteins were assessed using antibodies against p53, bax and bcl-2. GAPDH was used as an internal control. Relative band intensities were used for the quantification of (B) p53, (C) bax, (D) bcl-2 and (E) bax/bcl-2 ratios. ${ }^{*} \mathrm{P}<0.05 ;{ }^{* *} \mathrm{P}<0.01$. bcl-2, B-cell lymphoma 2; bax, bcl-2-associated $\mathrm{X}$.

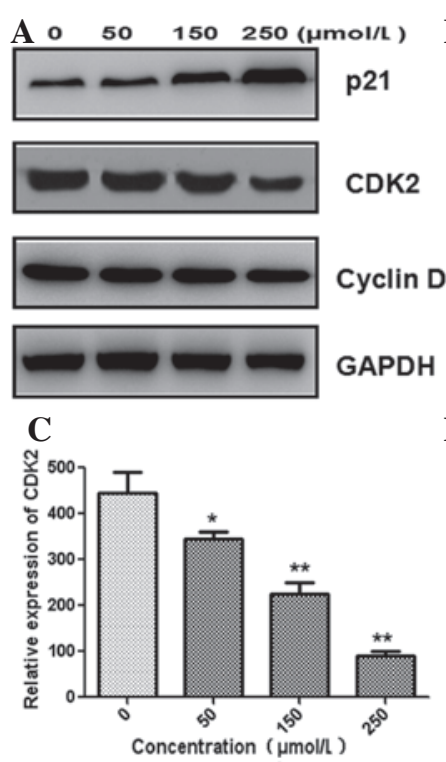

B

Figure 5. Effect of germacrone on the expression of cell cycle-associated proteins. Cells were incubated with germacrone at different concentrations for $24 \mathrm{~h}$ followed by western blot analysis. (A) Expression levels of cell cycle-associated proteins were assessed using antibodies against p21, CDK2 and cyclin D1. GAPDH was used as an internal control. The expression of (B) $\mathrm{p} 21,(\mathrm{C}) \mathrm{CDK} 2$ and (D) cyclin D was measured by relative band intensities. ${ }^{*} \mathrm{P}<0.05 ;{ }^{* *} \mathrm{P}<0.01$. CDK2, cyclin D kinase 2. 
G1 phase as compared with the control cells. The number of cells in $\mathrm{S}$ phase decreased compared with the levels in the controls (Fig. 3A and B). Similar results were observed in U251 cells following treatment with germacrone (Fig. 3C and D). These results demonstrated that germacrone induced cell cycle arrest in G1 phase in human glioma cells.

Effect of germacrone on the expression of apoptosis-and cell cycle-associated proteins. The expression levels of apoptosisand cell cycle-associated proteins were assessed using western blot analysis. The expression of tumor suppressor gene p53 was significantly increased (Fig. 4A and B). As shown in Fig. 4C and D, cells treated with germacrone showed an increase in bax expression and a decrease in bcl-2 expression in a dose-dependent manner. Densitometric analysis revelaed that the bax/bcl-2 ratio was significantly increased in a dose-dependent manner following treatment with germacrone (Fig. 4E). The expression of p21 was significantly increased following treatment with germacrone (Fig. 5A and B). The expression of G1-phase regulatory proteins, including CDK2 and cyclin D1, significantly decreased in a dose-dependent manner following treatment with germacrone for $24 \mathrm{~h}$ (Fig. 5C and D).

\section{Discussion}

Germacrone is a sesquiterpene derivative and has been shown to be one of the most promising agents against several types of cancer, including liver and breast cancer $(9,10)$. However, to the best of our knowledge, its effect on glioma and the underlying molecular mechanism has not been previously explored. In the present study, it was demonstrated for the first time that germacrone inhibited the proliferation of glioma cells by inducing cell cycle arrest in G1-phase and promoted apoptosis.

The MTT assay results revealed that germacrone exerted a significant inhibitory effect on the growth of the human glioma cell lines U87 and U251. However, no obvious effect on normal human astrocytes was observed following treatment with germacrone. Taken together, these data showed that germacrone inhibited the proliferation of glioma cells.

Apoptosis is the process of programmed cell death characterized by typical cellular and molecular features, including shrinkage, externalization of phosphatidylserine and condensation of chromatin (11). The present study has demonstrated that germacrone treatment promoted apoptosis of U87 and U251 as shown by flow cytometry, as well as DNA fragmentation. Cell cycle is a regulated process controlled by cell cycle checkpoints which ensure the fidelity of cell division in various types of cells $(12,13)$. However, cell cycle arrest is able to be triggered by various exogenous and endogenous stimulating factors and results in the breakdown of cell division, cell death and/or apoptosis (14). In the present study, germacrone induced cell cycle arrest in G1 phase, indicating another possible mechanism by which germacrone inhibits the proliferation of glioma cells.

The molecular mechanism of G1 cell cycle arrest and apoptosis induced by germacrone in human glioma cells was further investigated. It is well known that the protein p53, encoded by the TP53 gene, has a crucial role in multi-cellular organisms, where it regulates apoptosis and the cell cycle (15). When a cell is exposed to damage, p53 is activated and inhibits various downstream target genes involved in apoptosis and cell cycle arrest, including bax, p53-upregulated modulator of apoptosis (PUMA), bcl-2 and p21 (16). Bax is a p53 primary-response gene, which is involved in a p53-regulated pathway for the induction of apoptosis. p53 directly activates the proapoptotic protein bax, which permeabilizes mitochondria and engages in the apoptotic program (17). p53 also accumulates in the cytosol to activate the expression of bax and subsequently triggers apoptosis (18). The anti-apoptotic protein bcl-2 has been shown to prevent the disruption of mitochondrial physiology and block the release of cytochrome $\mathrm{C}$ from mitochondria, which is a response gene of p53 and involved in p53-regulated apoptosis (19). In the present study, germacrone treatment resulted in an increase in p53 and bax expression and a decrease in bcl-2 in a dose-dependent manner. The bax/bcl-2 ratio was significantly increased in a dose-dependent manner following treatment with germacrone. These results showed that germacrone promoted apoptosis of glioma cells.

The protein p21 is a tumor suppressor and acts as an inhibitor of cell cycle progression. It negatively regulates the G1/S phase transition and its up-regulation results in cell cycle arrest in G1 phase (20). In association with CDK2 complexes, it inhibits kinase activity and blocks cell cycle progression through G1/S arrest (21). CDK2 is a member of the cyclin-dependent kinase family of Ser/Thr protein kinases and is essential for the G1/S transition (22). The central role of CDK2 in the regulation of the cell cycle makes it a promising indicator for studying inhibitory molecules that are able to modify the degree of cell proliferation. CDK2 has been shown to be a crucial regulator of S-phase progression and was evaluated as an anticancer drug target (23). Cyclin D1 promotes progression through the G1-S phase of the cell cycle and the over-expression of cyclin D1 has a pivotal role in the development of numerous cancers, including breast, colon and prostate cancer, as well as melanoma $(24,25)$. The present study has shown that the protein expression levels of cyclin D1 and CDK2 were significantly decreased, while p21 expression was significantly increased, suggesting that germacrone treatment induced cell cycle arrest in G1 phase.

In conclusion, results of the present study have demonstrated that germacrone inhibited the proliferation of glioma cells by regulating the expression of proteins associated with cell cycle arrest in G1 phase and apoptosis. Therefore, germacrone may be a novel potential drug for the treatment of gliomas in the future.

\section{References}

1. Wang Y, Zhou Z, Luo H, et al. Combination of tamoxifen and antisense human telomerase RNA inhibits glioma cell proliferation and anti-apoptosis via suppression of telomerase activity. Mol Med Rep 3: 935-940, 2010.

2. Krakstad C and Chekenya M: Survival signalling and apoptosis resistance in glioblastomas: opportunities for targeted therapeutics. Mol Cancer 9: 135, 2010.

3. Lefranc F, Facchini V and Kiss R: Proautophagic drugs: a novel means to combat apoptosis-resistant cancers, with a special emphasis on glioblastomas. Oncologist 12: 1395-1403, 2007.

4. Liu YH, Li ML, Hsu MY, et al: Effects of a Chinese Herbal Medicine, Guan-Jen-Huang (Aeginetia indica Linn.), on renal cancer cell growth and metastasis. Evid Based Complement Alternat Med 2012: 935860, 2012. 
5. Bamba Y, Yun YS, Kunugi A and Inoue H: Compounds isolated from Curcuma aromatica Salisb. inhibit human P450 enzymes. J Nat Med 65: 583-587, 2011.

6. Claeson P, Panthong A, Tuchinda P, et al: Three non-phenolic diarylheptanoids with anti-inflammatory activity from Curcuma xanthorrhiza. Planta Med 59: 451-454, 1993.

7. Matsuda H,Ninomiya K, Morikawa T and Yoshikawa M: Inhibitory effect and action mechanism of sesquiterpenes from Zedoariae Rhizoma on D-galactosamine/lipopolysaccharide-induced liver injury. Bioorg Med Chem Lett 8: 339-344, 1998.

8. Morikawa T, Matsuda H, Ninomiya K and Yoshikawa M Medicinal foodstuffs. XXIX. Potent protective effects of sesquiterpenes and curcumin from Zedoariae Rhizoma on liver injury induced by D-galactosamine/lipopolysaccharide or tumor necrosis factor-alpha. Biol Pharm Bull 25: 627-631, 2002.

9. Liu Y, Wang W, Fang B, et al: Anti-tumor effect of germacrone on human hepatoma cell lines through inducing G2/M cell cycle arrest and promoting apoptosis. Eur J Pharmacol 698: 95-102, 2013.

10. Zhong Z, Chen X, Tan W, et al: Germacrone inhibits the proliferation of breast cancer cell lines by inducing cell cycle arrest and promoting apoptosis. Eur J Pharmacol 667: 50-55, 2011.

11. Pang M, Wang ZL, Gao CL, Qu P and Li HD: Characterization of apoptotic changes induced by yessotoxin in the Bel7402 human hepatoma cell line. Mol Med Rep 4: 547-552, 2011.

12. Gutierrez GJ, Tsuji T, Cross JV, et al: JNK-mediated phosphorylation of $\mathrm{Cdc} 25 \mathrm{C}$ regulates cell cycle entry and G(2)/M DNA damage checkpoint. J Biol Chem 285: 14217-14228, 2010.

13. Weinert $T$ and Hopper AK: tRNA traffic meets a cell-cycle checkpoint. Cell 131: 838-840, 2007.

14. Tanaka H, Arakawa H, Yamaguchi T, et al: A ribonucleotide reductase gene involved in a p53-dependent cell-cycle checkpoint for DNA damage. Nature 404: 42-49, 2000.

15. Hu W, Ge Y, Ojcius DM, et al: p53 signalling controls cell cycle arrest and caspase-independent apoptosis in macrophages infected with pathogenic Leptospira species. Cell Microbiol 15 : $1642-1659,2013$
16. Yan X, Feng J, Ye Z, Miao X, Li W and Yang D: p53 siRNA inhibits apoptosis of U2OS cells treated with azurin. Mol Med Rep 4: 1089-1094, 2011

17. Deng Y and Wu X: Peg3/Pw1 promotes p53-mediated apoptosis by inducing Bax translocation from cytosol to mitochondria. Proc Natl Acad Sci USA 97: 12050-12055, 2000.

18. Gogada R, Prabhu V, Amadori M, Scott R, Hashmi S and Chandra D: Resveratrol induces p53-independent, X-linked inhibitor of apoptosis protein (XIAP)-mediated Bax protein oligomerization on mitochondria to initiate cytochrome c release and caspase activation. J Biol Chem 286: 28749-28760, 2011.

19. Tsutsui S, Yasuda K, Suzuki K, et al: Bcl-2 protein expression is associated with p27 and p53 protein expressions and MIB-1 counts in breast cancer. BMC Cancer 6: 187, 2006.

20. Pirngruber J and Johnsen SA: Induced G1 cell-cycle arrest controls replication-dependent histone mRNA 3' end processing through p21, NPAT and CDK9. Oncogene 29: 2853-2863, 2010.

21. Satyanarayana A, Hilton MB, and Kaldis P: p21 Inhibits Cdk1 in the absence of Cdk2 to maintain the G1/S phase DNA damage checkpoint. Mol Biol Cell 19: 65-77, 2008.

22. Li W, Kotoshiba S, Berthet C, Hilton MB and Kaldis P: $\mathrm{Rb} / \mathrm{Cdk} 2 / \mathrm{Cdk} 4$ triple mutant mice elicit an alternative mechanism for regulation of the G1/S transition. Proc Natl Acad Sci USA 106: 486-491, 2009

23. Chen XZ, Cao ZY, Chen TS, et al: Water extract of Hedyotis Diffusa Willd suppresses proliferation of human HepG2 cells and potentiates the anticancer efficacy of low-dose 5-fluorouracil by inhibiting the CDK2-E2F1 pathway. Oncol Rep 28: 742-748, 2012.

24. Fang Y, Cao Z, Hou Q, et al: Cyclin D1 downregulation contributes to anticancer rffect of isorhapontigenin on human bladder cancer cells. Mol Cancer Ther 12: 1492-1503, 2013.

25. Grillo M, Bott MJ, Khandke N, et al: Validation of cyclin D1/CDK4 as an anticancer drug target in MCF-7 breast cancer cells: Effect of regulated overexpression of cyclin D1 and siRNA-mediated inhibition of endogenous cyclin D1 and CDK4 expression. Breast Cancer Res Treat 95: 185-194, 2006. 